\title{
A propensity score matching study of the short-term efficacy of azygos arch-sparing McKeown minimally invasive esophagectomy
}

\author{
Hui Li ${ }^{1}$, Jiongzhen Lin ${ }^{2}$, Hai Zhang', Bomeng $\mathrm{Wu}^{2}$, Ying Chen ${ }^{2}$, Haiquan $\mathrm{He}^{2}$, Linrong Zhou ${ }^{2}$, \\ Lanjuan Gong', Gang Chen ${ }^{3}$, Maosheng Wang ${ }^{2}$, Wanli Lin ${ }^{2}$ \\ ${ }^{1}$ Department of Thoracic Surgery, Zhujiang Hospital, Southern Medical University, Guangzhou, China; ${ }^{2}$ Department of Thoracic Surgery, Gaozhou \\ People's Hospital, Guangdong Esophageal Cancer Institute Gaozhou Branch, Gaozhou, China; ${ }^{3}$ Department of Thoracic Surgery, Guangdong \\ Provincial People's Hospital, Guangdong Academy of Medical Sciences, Guangzhou, China \\ Contributions: (I) Conception and design: W Lin; (II) Administrative support: M Wang; (III) Provision of study materials or patients: J Lin, H He; (IV) \\ Collection and assembly of data: J Lin, H Zhang, H He, L Zhou, L Gong; (V) Data analysis and interpretation: H Li, J Lin, B Wu, Y Chen, G Chen; \\ (VI) Manuscript writing: All authors; (VII) Final approval of manuscript: All authors. \\ Correspondence to: Wanli Lin; Maosheng Wang. Department of Thoracic Surgery, Gaozhou People’s Hospital, Guangdong Esophageal Cancer \\ Institute Gaozhou Branch, Gaozhou 525200, China. Email: wanlilin2020@163.com; wangmaosheng_123@163.com.
}

\begin{abstract}
Background: To evaluate the short-term efficacy of azygos arch-sparing McKeown minimally invasive esophagectomy (McKeown-MIE).

Methods: We retrospectively analyzed the clinical data of 221 patients with thoracic esophageal squamous cell carcinoma who underwent McKeown-MIE at the Department of Thoracic Surgery of Gaozhou People's Hospital from August 1, 2017 to September 30, 2019. According to whether the azygos arch was preserved or not, the patients were assigned to one of two groups: the preservation group (40 cases) and the ligation group (181 cases). Within 3 months of the operation, the perioperative outcomes and the postoperative short-term efficacy of the two groups were compared.

Results: After propensity score (PS) matching, 40 pairs of patients were matched successfully. Between the two groups, there were no statistical difference in intraoperative blood loss, the number of lymph nodes dissected, thoracic drainage duration, fasting time, postoperative hospital stay time, and major postoperative complications $(\mathrm{P}>0.05)$. Compared with the ligation group, patients in the preservation group had a shorter intensive care unit (ICU) stay time, a shorter operative time, a lower volume of postoperative thoracic drainage (both the first 3 days and overall) following surgery, a tubular stomach that had a smaller caliber, and a lower incidence of tubular gastric malpositioning $(\mathrm{P}<0.05)$.

Conclusions: Preserving the azygos arch during a McKeown-MIE is safe and feasible. Doing so, not only effectively restricts the expansion of the gastric conduit, leading to a lower incidence of malpositioning, but also dramatically reduces postoperative thoracic drainage, and ICU stay time.
\end{abstract}

Keywords: Esophagectomy; esophageal squamous cell carcinoma; azygos vein

Submitted Dec 09, 2020. Accepted for publication Feb 08, 2021.

doi: 10.21037/jgo-21-14

View this article at: http://dx.doi.org/10.21037/jgo-21-14

\section{Introduction}

Esophageal cancer is one of the most common gastrointestinal tumors around the world (1). Radical resection is the preferred treatment for esophageal cancer. In recent years, due to the continuous improvement of endoscopic technologies, thoracoscopy- and laparoscopyassisted minimally invasive esophagectomy (MIE) has rapidly gained popularity around the globe. Of all the MIE approaches, the McKeown-MIE procedure is popular among many surgeons, as it allows for more complete lesion resection and thorough lymph node dissection $(2,3)$. 
However, during the McKeown-MIE procedure, the azygos arch is routinely ligated and severed to facilitate lymph node dissection and esophagus mobilization. During our work, as experience accumulated, we found that the azygos arch could be spared in many patients when the McKeownMIE procedure was performed, which had the potential advantages of maintaining the mediastinal venous reflux and reducing the tubular gastric expansion and malpositioning. To evaluate the short-term effectiveness of this modified McKeown-MIE procedure, we retrospectively analyzed the clinical data of 221 patients who received the McKeownMIE procedure in our department in the past two years by using the propensity score matching (PSM) method. The results provided some insights that will assist us in our future long-term study.

We present the following article in accordance with the STROBE reporting checklist (available at http://dx.doi. org/10.21037/jgo-21-14).

\section{Methods}

\section{Study participants}

The clinical data of 221 consecutive patients who received McKeown-MIE surgery at Thoracic Surgery Department of Gaozhou People's Hospital from August 1, 2017 to September 30, 2019 were retrospectively collected. All patients were diagnosed as having thoracic esophageal squamous cell carcinoma by pathological biopsy under endoscopy. A comprehensive preoperative evaluation consisting of a physical examination, contrast-enhanced computed tomography (CT) scans of the chest and abdomen, pulmonary function tests, electrocardiography, cardiac echocardiography, and a barium meal assessment was performed to assess the resectability of the patients' tumors. Based on clinical assessments, patients were included in the study if they met the following inclusion criteria: (I) had clinically resectable tumors located in the thoracic esophagus; (II) had not undergone neoadjuvant chemoradiotherapy; and (III) had no previous history of cancer. Based on clinical assessments, patients were excluded from the study if they met any of the following exclusion criteria: (I) had a previous history of cancer; (II) had heart, liver, renal, or diabetes dysfunction; (III) had had tumors palliatively resected intraoperatively; (IV) died within 3 months of the surgery or who were not followed up with postoperatively. The patients were divided into the modified McKeown-MIE group (preservation group) or the traditional McKeown-MIE group (ligation group) based on whether or not the azygos arch was preserved intraoperatively. In total, there were 40 patients in the preservation group and 181 patients in the ligation group. All procedures performed in this study involving human participants were in accordance with the Declaration of Helsinki (as revised in 2013). This study was approved by the Gaozhou People's Hospital Human Research Ethics Committee (No. GYLLPJ-2010064). Individual consent for this retrospective analysis was waived.

\section{Operative method}

\section{Thoracoscopic procedure}

In relation to the thoracoscopic procedure for patients in the ligation group, after general anesthesia, the patient was placed in the left lateral decubitus position and ventilated by a single lumen endotracheal intubation. The surgeon stood on the dorsal part of the patient. Four ports were made as follows: a $1 \mathrm{~cm}$ optical port was placed in the $6^{\text {th }}$ intercostal space at the anterior axillary line; a $1 \mathrm{~cm}$ utility port was placed in the $4^{\text {th }}$ intercostal space at the anterior axillary line; the other 0.5 and $1 \mathrm{~cm}$ utility ports were placed in scapular line at the $7^{\text {th }}$ and $9^{\text {th }}$ intercostal spaces, respectively. After inspection, the esophagus was mobilized from the hiatus to the thoracic inlet. The azygos vein was routinely transected, and lymph nodes were removed at the paratracheal, subcarinal, and paraoesophageal areas, including both sides of the recurrent laryngeal nerve (RLN). In relation to the thoracoscopic procedure for patients in the preservation group, the method of anesthesia and the ports placed were the same as those for patients in the litigation group. The esophagus was then dissociated from the azygos vein with the azygos arch and the right bronchial artery separated and preserved without resection. The scope of the lymph node dissection was performed routinely as described above.

\section{The cervical and abdominal procedure}

After completing the thoracoscopic procedure, the patient was rotated to a supine position, with the neck extended and turned to the right. An approximate $5 \mathrm{~cm}$ oblique incision was made over the anterior border of the left sternocleidomastoid muscle. The cervical esophagus was mobilized and transected, the proximal end was then inserted into a pedestal of the circular stapler device and the distal end of the esophagus was ligated and connected with a $50 \mathrm{~cm}$ long $16 \mathrm{~F}$ rubber tube to assist with the 
lifting of the gastric conduit. After the cervical procedure, pneumoperitoneum was established with $10-12 \mathrm{mmHg}$ with carbon dioxide, and five abdominal ports were then made routinely. The stomach was dissected free with preservation of the right gastroepiploic vessels and the mobilized thoracic esophagus with the distal end of the rubber tube was pulled into the abdominal cavity. After the gastric mobilization for the conduit, a complete abdominal lymphadenectomy, including of the celiac axis, common hepatic, left gastric and distal esophagus, was performed. The then mobilized stomach was pulled out from the extended incision below the xiphoid process, and a $3-\mathrm{cm}$ wide gastric conduit was molded with the linear stapler, which was then sutured in an uninterrupted fashion and reconnected with the distal end of the rubber tube. After the esophagus, the tumor and the fundus of the stomach were removed, the gastric conduit was put back in situ. Ultimately, the gastric conduit was pulled up to the left cervical incision through the posterior mediastinum, assisted by the rubber tube, and a cervical end-to-side anastomosis was then constructed at the high point of the gastric conduit using a circular stapler. The distal end of the gastric tube was closed using a linear stapler approximately $2-3 \mathrm{~cm}$ away from the circular anastomosis. After the anastomotic site was embedded, the cervical and abdominal incisions were closed layer by layer, and the operation was completed.

\section{Observation indexes}

Perioperative observation indexes included operative time, intraoperative blood loss, tumor length, and the number of lymph nodes dissected. Postoperative indicators included postoperative thoracic drainage volume, chest drainage time, ICU stay time, postoperative fasting time, postoperative hospital stay time, the incidence of postoperative complications (including pneumothoraxes, swallowing dysfunction, anastomotic and residual stomach fistulas, pulmonary infections, chylothoraxes, upper gastrointestinal hemorrhages, respiratory failure, and pulmonary embolisms). The short-term follow-up indexes at 3 months after the operation included tubular gastric diameter and the incidence of poor tubular gastric positioning and anastomotic stenosis. The postoperative maximal luminal diameter of the tubular gastric tube was measured at the level of azygos arch by CT. The malpositioning of the tubular stomach was defined as an obvious distortion or displacement of the stomach conduit that was not confined to the mediastinal esophageal bed and compressed the surrounding thoracic structures, such as the lungs, the heart or the mediastinum. The anastomotic stricture was regarded as dysphagia in combination with a luminal diameter of $\leq 8 \mathrm{~mm}$ at esophagogram. All the postoperative complications were recorded within 2 weeks of surgery.

\section{Statistical analysis}

IBM SPSS Statistics 25.0 statistical software was used. Patients' age, tumor length, the number of intraoperative lymph nodes dissected, operative time, intraoperative blood loss, thoracic drainage volume in the first 3 days after surgery, total thoracic drainage volume, duration of thoracic drainage, ICU stay time, postoperative fasting time, postoperative hospital stay, and tube gastric diameter were all showed as mean \pm standard deviation $(\bar{x} \pm \mathrm{s})$. The differences between the two groups were compared using the Student's $t$-test. The preoperative level of pulmonary dysfunction, tumor grade (G) stage, primary tumor (T) stage, regional lymph nodes $(\mathrm{N})$ stage, and pathological stage [according to the The American Joint Committee on Cancer/Union Internationale Contre le Cancer (AJCC/UICC) cancer stages in the 8th edition (4)] of the two groups were expressed as percentages, and a KruskalWallis $\mathrm{H}$ test was conducted to compare the two groups. Gender, basic diseases, intraoperative thoracic duct ligation, tumor location, major postoperative complications, anastomotic stenosis, poor tubular stomach position, and other qualitative data of the two groups were expressed as percentages, and a Chi-square test was used to compare the two groups. The 13 parameters of patient age, gender, history of heart disease, hypertension and diabetes, lung ventilation function obstacle degree, $\mathrm{G}$ stage, $\mathrm{T}$ stage, $\mathrm{N}$ stage, pathological stage, tumor location, tumor length and ligation of thoracic duct served as concomitant variables for PSM, and the calipers value was 0.06. Additionally, the propensity score (PS) was calculated using a logistic regression formula. The PS value and PS weight were then obtained, and random matching was carried out in a 1:1 ratio. The statistics for all the variables were analyzed. A P value of $<0.05$ was considered to be statistically significant.

\section{Results}

\section{General information of patients in both groups}

As stated above, there were 40 patients in the preservation 
group and 181 patients in the ligation group. A total of 40 pairs of patients were successfully matched after PSM with a 1:1 ratio. There was no statistical significance in the age, gender, history of heart disease, history of hypertension, history of diabetes, degree of pulmonary dysfunction, $G, T$, $\mathrm{N}$, or $\mathrm{M}$ stage, pathological stage, tumor location, tumor length, and thoracic duct ligation of patients between two groups before or after PSM (see Tables 1 and 2).

\section{Perioperative indicators}

Compared with that in the ligation group, sparing the azygos arch did not prolong the operative time in the preservation group $[(255.23 \pm 46.90) v s$. $(307.39 \pm 47.51) \mathrm{min}$, $t=-5.182, \mathrm{P}<0.001]$. Further, the postoperative ICU stay time $[(19.06 \pm 11.48) v s .(27.21 \pm 21.74) \mathrm{h}, t=-2.097, \mathrm{P}=0.039]$ was shorter in the preservation group than that in the ligation group. Notably, the thoracic drainage volume within 3 days of the operation was $469.25 \pm 312.58 \mathrm{vs}$. $786.95 \pm 400.12 \mathrm{~mL}(t=-3.957, \mathrm{P}<0.001)$, and the total thoracic drainage volume was obviously less in the preservation group. Other indexes between the two groups, such as the intraoperative blood loss, number of lymph nodes dissected, thoracic drainage time, postoperative fasting time, postoperative hospital stay time, and the major postoperative complications, had no statistical significance $(\mathrm{P}>0.05)$ (see Table 3).

\section{Postoperative short-term follow-up indexes}

There were no deaths within 3 months of the operation in either group and no obvious thoracic stomach syndrome was observed. The maximal diameter of the tubular stomach $[(14.50 \pm 6.29) v s .(26.41 \pm 10.90) \mathrm{mm}, t=-5.986, \mathrm{P}<0.001]$ and the percentage of the malposition of the tubular stomach ( $7.5 \%$ vs. $\left.50.0 \%, \chi^{2}=17.635, \mathrm{P}<0.001\right)$ were less in the preservation group (see Figures 1 and 2). The incidence of postoperative anastomotic stricture between the two groups was not statistically significant $(\mathrm{P}>0.05)$ (see Table 3).

\section{Discussion}

Esophageal cancer (EC) is among the top causes of cancerrelated mortality globally, resulting in more than 400 thousand deaths each year and half of them occur in China (5). The primary therapeutic method for esophageal cancer has been surgical resection. However, the traditional open esophagectomy is associated with significant morbidity and mortality. As a result, the minimally invasive esophagectomy (MIE) under thoracoscopy and laparoscopy was developed, and the safety and feasibility of MIE are well-recognized, which are attributed to be superior than open esophagectomy in reducing postoperative outcomes, without compromising oncological outcomes and avoiding thoracotomy and laparotomy (6). Now, the MIE comprised two main surgical approaches: McKeown-MIE approach (cervical anastomosis) and Ivor-Lewis- MIE approach (intrathoracic anastomosis). Compared with the IvorLewis procedure in which an intrathoracic esophagogastric anastomosis is performed after the tumors in the middlelower esophagus are resected, the McKeown procedure is applicable to all esophageal cancers below the clavicle level, and has a number of potential advantages, including a reduced chance of local recurrence, a wider range of lymph node dissection, and the easier management of anastomosis if a leak occurs (7).

The azygos arch is an important adjacent structure of the esophagus, which collects a large amount of mediastinal blood and lymph from the partial collateral of the thoracic duct. However it is routinely ligated and severed during the McKeown-MIE procedure to assist with the esophageal dissociation, and the dissection of the left recurrent laryngeal nerve lymph nodes, which may hinder the mediastinal venous reflux, increase the volume of pleural effusion and aggravate tissue edema (8-10). Additionally, the dissected venous arch loses its anatomical "banding" effect on the esophagus. After being pulled upward from the abdomen to the neck, due to the destruction of the gastric wall, the denervation of the vagus nerve and the negative pressure of the thoracic cavity, the tubular stomach usually has varying degrees of expansion or dilation, which may cause gastric retention, respiratory aspiration, and even thoracic stomach syndrome. Further, the expanded conduit may affect the blood supply of the gastric surgical margin, adding the potential risks of gastric fistula or anastomosis fistula $(11,12)$. In the present study, the thoracic drainage volume in the first three days and the total thoracic drainage volume in the preservation group were significantly less than that in the ligation group, which may be attributable to the sparing of the azygos arch. Additionally, the preservation group had a smaller caliber of tubular stomach and a lower incidence of tubular gastric malposition compared to that in the ligation group $[(14.50 \pm 6.29)$ vs. $(26.41 \pm 10.90) \mathrm{mm}$, $\mathrm{P}<0.001,50 \%$ vs. $7.5 \%(\mathrm{P}<0.001)]$, which appears to be due to the "banding" property of the preserved vein arch.

Moreover, sparing the azygos vein arch did not 
Table 1 A comparison of the general data of patients in the two groups before PSM

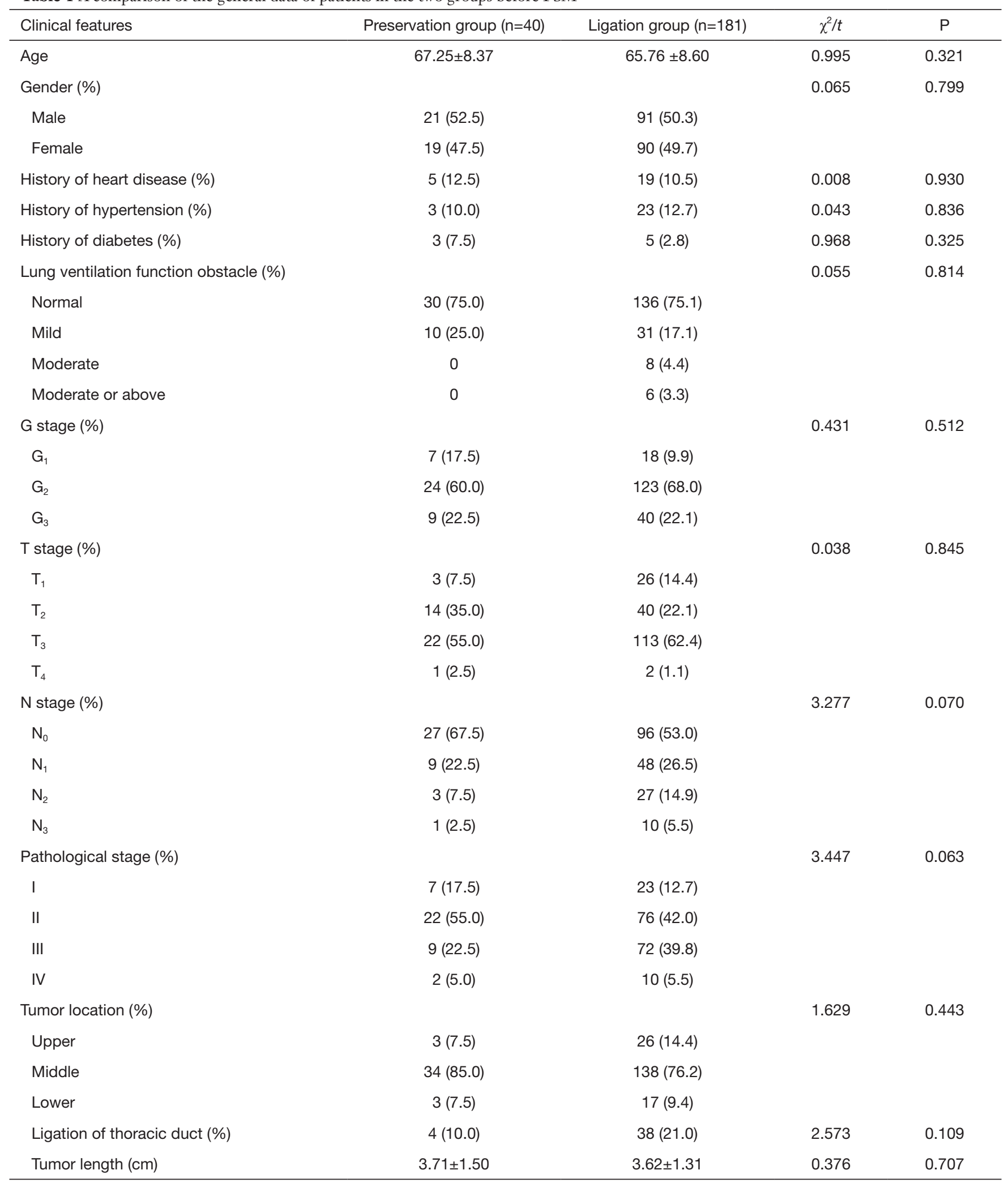

PSM, propensity score matching. 
Table 2 Comparison of general data of patients in two groups after PSM

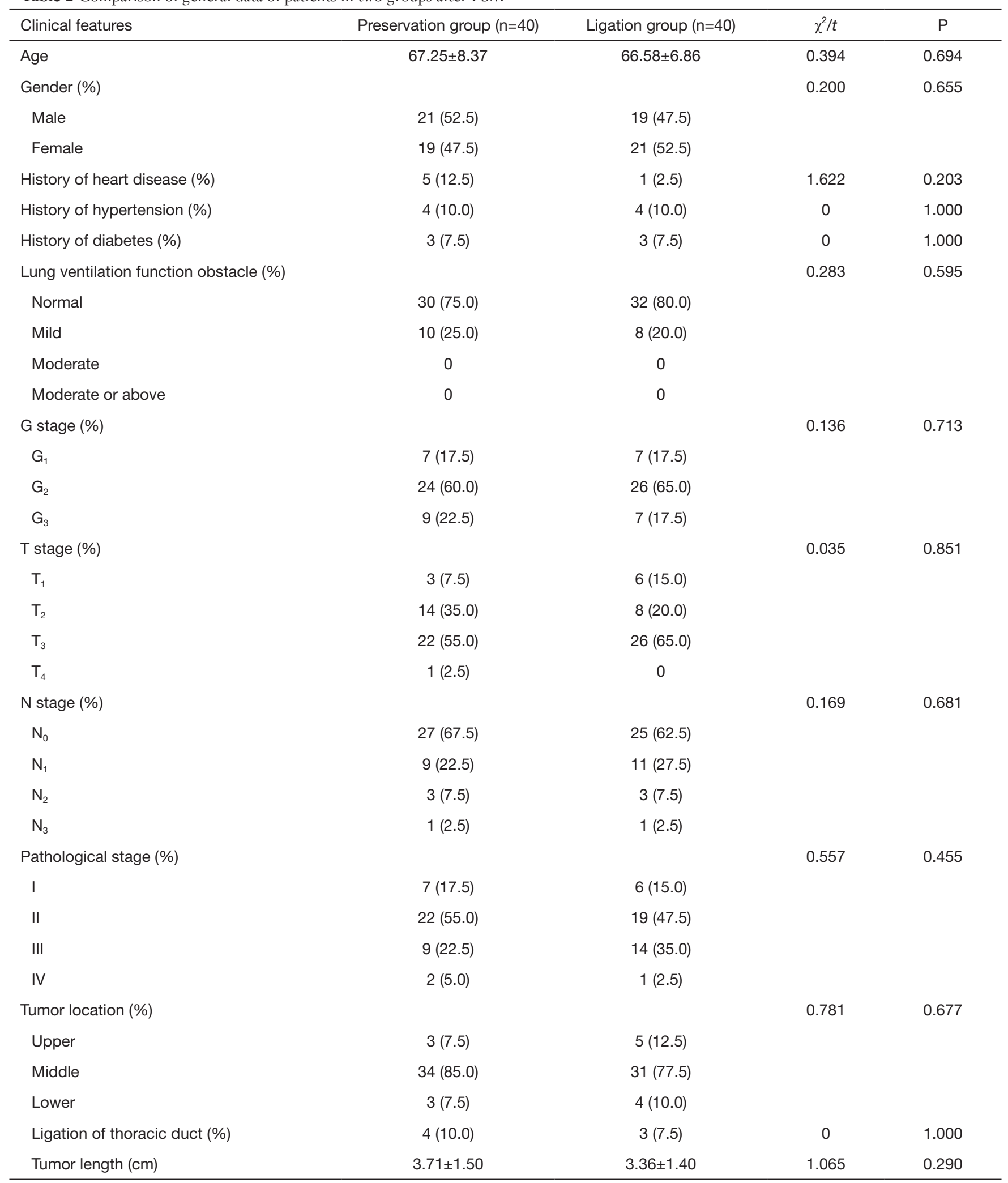

PSM, propensity score matching. 
Table 3 Comparison of intraoperative and postoperative data of patients in two groups after PSM

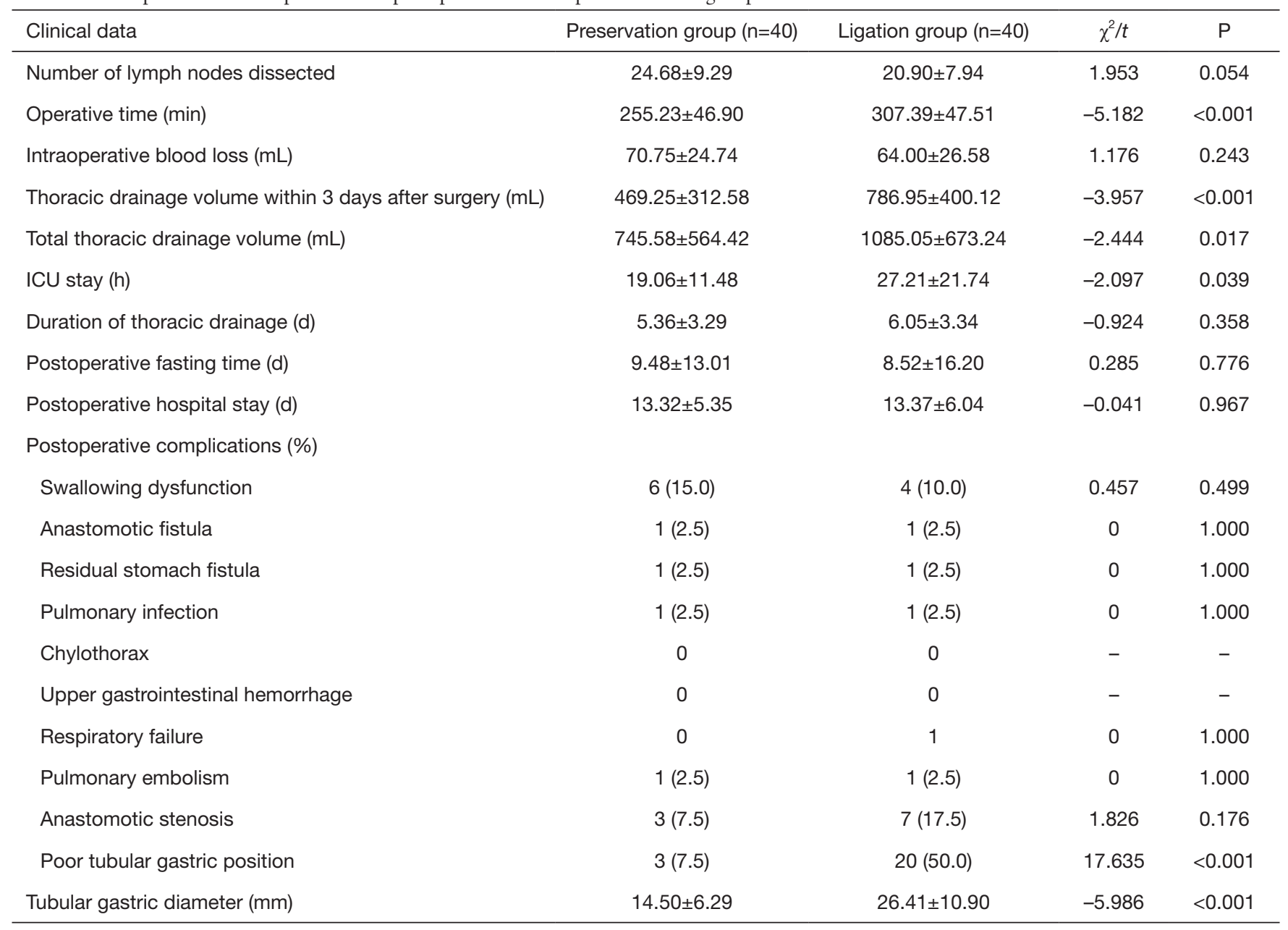

PSM, propensity score matching.

complicate lymph node dissection, and there was no statistical difference in the number of lymph node dissections between the preservation group and the ligation group (24.68 $\pm 9.29 v s .20 .90 \pm 7.94, t=1.953 \mathrm{P}=0.054)$. It should also be noted that bronchial artery preservation has been suggested as a method to reduce tracheal ischemia, which contributes to pulmonary complications because of tracheal erosions, ulcerations, and reduced mucus secretion (13). Anatomically, the right bronchial artery and the azygos arch are usually connected as a whole, with the former entering the right hilum just beneath the latter. Thus, most of the right bronchial arteries of the patients in the preservation group were also retained. However, there was no statistically significant difference between the two groups in terms of the incidence of pulmonary infection, respiratory failure, and other complications. This may be due to the small sample size of the study, and the limited observed indicators of pulmonary complications. In the future, we intend to explore the efficacy of preserving the right bronchial artery in this modified McKeown-MIE procedure.

In terms of safety, there was no statistically significant difference in the incidence of postoperative major complications, such as anastomotic fistulas, residual gastric fistulas, swallowing dysfunction, anastomotic stenoses, chylothoraxes, pulmonary infections, pulmonary embolisms, respiratory failure, and upper gastrointestinal bleeding, between the preservation group and the ligation group $(\mathrm{P}>0.05)$. Additionally, the postoperative ICU stay time in the preservation group was shorter than that in the ligation group, which may fast track the recovery of patients. However, it should be noted that this modified azygos archsparing procedure will not be suitable for all patients. For 

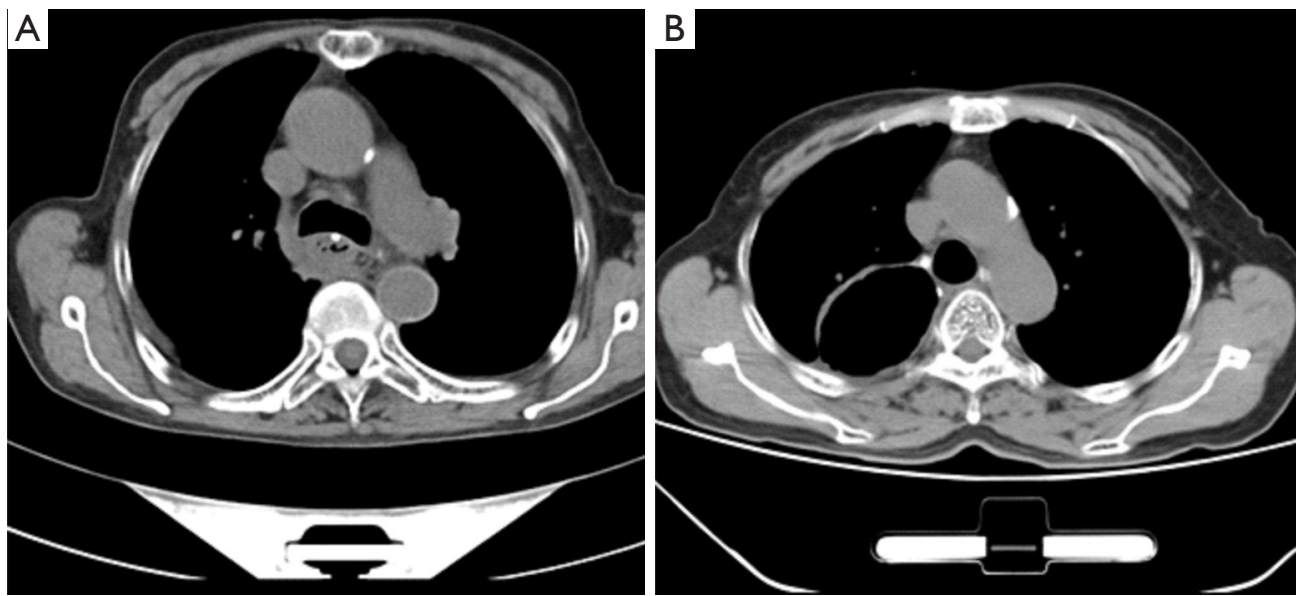

Figure 1 The diameter of the thoracic stomach conduit in the preservation group (left) was smaller than that in the ligation group (right) at the level of the azygos vein plane as shown in transverse view of the chest CT at 3 months postoperatively.
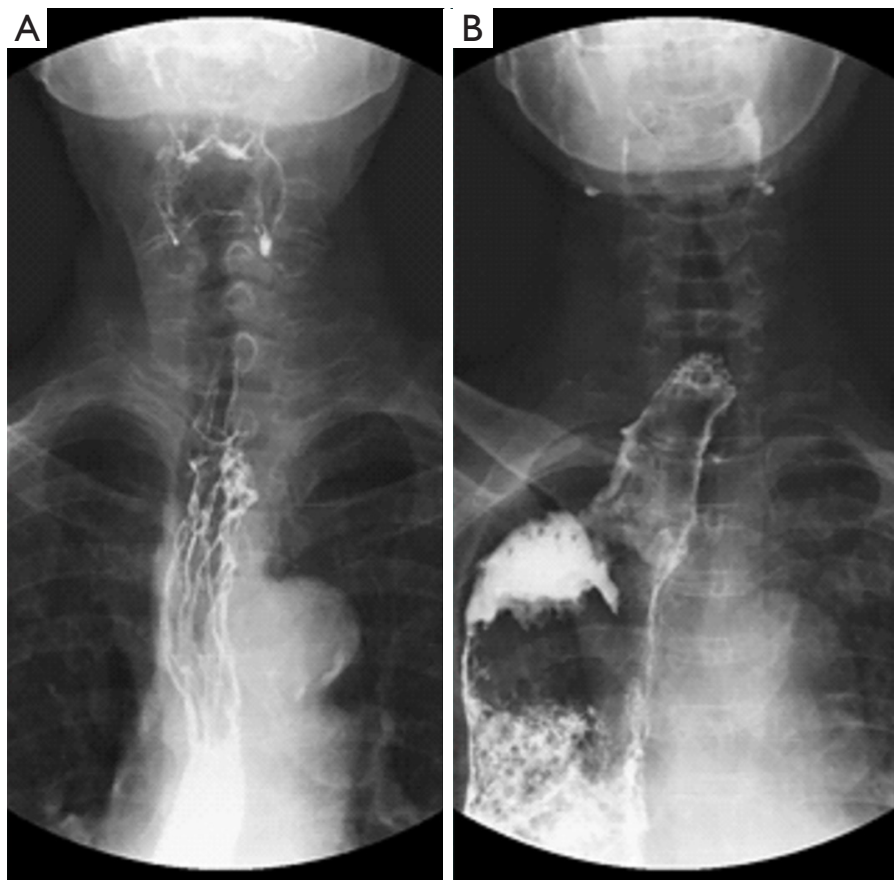

Figure 2 Esophagograms 3 months after the operation showed the gastric conduit in the preservation group was well confined to the mediastinum (left), which contrasted sharply to the dilated gastric tube in the ligation group (right).

patients with large tumors located near the azygos arch or with tumors invading the azygos arch, the vein arch should be resected routinely. In the ligation group, the azygos arch was resected largely due to the anatomic difficulty of dissecting the esophagus from the arch, which may also explain why the operative time in the preservation group was shorter than that in the ligation group. Based on our practice, before the tubular conduit was put back in situ, it should be carefully examined for any left residue or poorly formed stapler nails. Further, when the conduit was lifted upward through the azygos arch, the anesthesiologist is usually directed to temporarily stop the ventilation, which may facilitate the lifting of the conduit and reduce the possibility of azygos arch tearing. In our study, no 
intraoperative bleeding occurred in the 40 patients in the preservation group.

In this study, PSM was used to balance the distribution of various clinical characteristics between the preservation group and the ligation group to improve the reliability of our results. However, this study had some limitations. First, it was not a randomized multi-center prospective clinical study. Second, the observation period was short, and we did not evaluate the significance of this modified McKeown-MIE in terms of the long-term postoperative effects. Third, the observational indicators of the study are limited. Patients who undergo esophagectomy with gastric conduit reconstruction are at risk of developing functional disorders, such as delayed gastric emptying, dumping syndrome, reflux, and dysphagia, which sometimes can seriously affect patients' quality of life. The reduced diameter and malposition rate of the gastric conduit may contribute to the controlling of these functional disorders. Thus, more research needs to be conducted in the future to further analyze the significance of the azygos arch-sparing technique on the long-term postoperative course.

In conclusion, the results of the present study showed that azygos arch sparing during the McKeown-MIE procedure is safe and effective. The preservation of the azygos arch did not increase the incidence of postoperative complications, and also contributed to a shortened postoperative ICU stay, and reduced postoperative thoracic drainage, which appear to speed up the recovery of the patients. Additionally, the "banding" function of the spared azygos arch may help to constrict the dilatation of the gastric tube and reduce the incidence of poor tubular stomach positioning. This appears to be the first report of the short-term efficacy of the azygos arch-sparing McKeown-MIE procedure. Given the observed positive short-term effects, we intend to explore the long-term efficacy of this procedure further.

\section{Acknowledgments}

Funding: None.

\section{Footnote}

Reporting Checklist: The authors have completed the STROBE reporting checklist. Available at http://dx.doi. org/10.21037/jgo-21-14

Data Sharing Statement: Available at http://dx.doi. org/10.21037/jgo-21-14

Conflicts of Interest: All authors have completed the ICMJE uniform disclosure form (available at http://dx.doi. org/10.21037/jgo-21-14). The authors have no conflicts of interest to declare.

Ethical Statement: The authors are accountable for all aspects of the work in ensuring that questions related to the accuracy or integrity of any part of the work are appropriately investigated and resolved. All procedures performed in this study involving human participants were in accordance with the Declaration of Helsinki (as revised in 2013). This study was approved by the Gaozhou People's Hospital Human Research Ethics Committee (No. GYLLPJ-2010064). Individual consent for this retrospective analysis was waived.

Open Access Statement: This is an Open Access article distributed in accordance with the Creative Commons Attribution-NonCommercial-NoDerivs 4.0 International License (CC BY-NC-ND 4.0), which permits the noncommercial replication and distribution of the article with the strict proviso that no changes or edits are made and the original work is properly cited (including links to both the formal publication through the relevant DOI and the license). See: https://creativecommons.org/licenses/by-nc-nd/4.0/.

\section{References}

1. Bray F, Ferlay J, Soerjomataram I, et al. Global cancer statistics 2018: GLOBOCAN estimates of incidence and mortality worldwide for 36 cancers in 185 countries. CA Cancer J Clin 2018;68:394-424.

2. Deng J, Su Q, Ren Z, et al. Comparison of short-term outcomes between minimally invasive McKeown and Ivor Lewis esophagectomy for esophageal or junctional cancer: a systematic review and meta-analysis. Onco Targets Ther 2018;11:6057-69.

3. Brown AM, Pucci MJ, Berger AC, et al. A standardized comparison of peri-operative complications after minimally invasive esophagectomy: Ivor Lewis versus McKeown. Surg Endosc 2018;32:204-11.

4. Rice TW, Ishwaran H, Ferguson MK, et al. Cancer of the Esophagus and Esophagogastric Junction: An Eighth Edition Staging Primer. J Thorac Oncol 2017;12:36-42.

5. Hou H, Meng Z, Zhao X, et al. Survival of Esophageal Cancer in China: A Pooled Analysis on Hospital-Based 
Studies From 2000 to 2018. Front Oncol 2019;27;9:548.

6. Yibulayin W, Abulizi S, Lv H, Sun W. Minimally invasive oesophagectomy versus open esophagectomy for resectable esophageal cancer: a meta-analysis. World J Surg Oncol 2016;14:304.

7. van Workum F, Berkelmans GH, Klarenbeek BR, et al. McKeown or Ivor Lewis totally minimally invasive esophagectomy for cancer of the esophagus and gastroesophageal junction: systematic review and metaanalysis. J Thorac Dis 2017;9:S826-33.

8. Cui X, He Y, Chen L, et al. Clinical Analysis of Azygos Vein Preservation Under Thoracoscope in the Operation of Type III Esophageal Atresia. J Laparoendosc Adv Surg Tech A 2020;30:448-52.

9. Sharma S, Sinha SK, Rawat JD, et al. Azygos vein preservation in primary repair of esophageal atresia with

Cite this article as: $\mathrm{Li} \mathrm{H}$, Lin J, Zhang $\mathrm{H}, \mathrm{Wu} \mathrm{B}$, Chen Y, He H, Zhou L, Gong L, Chen G, Wang M, Lin W. A propensity score matching study of the short-term efficacy of azygos arch-sparing McKeown minimally invasive esophagectomy. J Gastrointest Oncol 2021;12(1):28-37. doi: 10.21037/jgo-21-14 tracheoesophageal fistula. Pediatr Surg Int 2007;23:1215-8.

10. Upadhyaya VD, Gangopadhyaya AN, Gopal SC, et al. Is ligation of azygos vein necessary in primary repair of tracheoesophageal fistula with esophageal atresia? Eur J Pediatr Surg 2007;17:236-40.

11. Konradsson M, Nilsson M. Delayed emptying of the gastric conduit after esophagectomy. J Thorac Dis 2019;11:S835-44.

12. Meyerson SL, Mehta CK. Managing complications II: conduit failure and conduit airway fistulas. J Thorac Dis 2014;6 Suppl 3:S364-71.

13. Pramesh CS, Mistry RC, Sharma S, et al. Bronchial artery preservation during transthoracic esophagectomy. J Surg Oncol 2004;85:202-3.

(English Language Editor: L. Huleatt) 